\title{
A Culturally Appropriate Educational Intervention Can Improve Self-Care in Hispanic Patients With Heart Failure: A Pilot Randomized Controlled Trial
}

\author{
Jill Howie-Esquivel ${ }^{\mathrm{a}, \mathrm{e}}$, Kirsten Bibbins-Domingo ${ }^{\mathrm{b}}$, Robyn Clark ${ }^{\mathrm{c}}$, \\ Lorraine Evangelista ${ }^{d}$, Kathleen Dracup ${ }^{\mathrm{a}}$
}

\begin{abstract}
Background: Hispanics constitute the largest US ethnic group and have been shown to have more frequent heart failure (HF) hospitalizations than non-Hispanic whites. Disease management programs can reduce HF hospitalizations and mortality by increasing patient self-care, but most programs are limited to patients who speak English. Therefore, we hypothesize that Project Fluido, a culturally appropriate self-care education intervention, will improve self-care behaviors and knowledge in Hispanic patients with HF compared with usual care (UC).
\end{abstract}

Methods: Project Fluido $(\mathrm{N}=42)$ was a randomized controlled pilot trial over 3 months. Patients in the experimental group $(\mathrm{n}=$ 22) received individualized education in Spanish using the "teachback" method on the following: high salt foods, when to call the physician, when to report weight gain and the use of diuretics. They also received a nurse-initiated phone call every 2 weeks, a script for calling their physician with increased symptoms, a weight scale and a daily diary to complete. The UC group $(n=20)$ received a scale and written information. Self-care was measured using the self-care heart failure index and knowledge using teach-back scores. Four knowledge topics were included when using teach-back.

Results: Participants' mean age was $57 \pm 14$ years, $57 \%$ (24) were male, 64\% (27) had hypertension, 86\% (36) were New York Heart Association Class I-III and 65\% (26) had HF with reduced ejection fraction. Participant health literacy scores showed poor health literacy in $31 \%(\mathrm{n}=13)$ and $67 \%(\mathrm{n}=28)$ spoke Spanish only. Household income was reported as $<\$ 20,000$ in $93 \%(n=39)$. Self-

Manuscript accepted for publication July 23, 2014

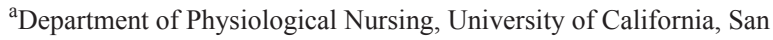
Francisco, CA, USA

${ }^{b}$ Department of Medicine, University of California, San Francisco General Hospital, San Francisco, CA, USA

${ }^{\mathrm{c} S}$ chool of Nursing and Midwifery, Flinders University, Adelaide, South Australia, Australia

${ }^{\mathrm{d}}$ Program in Nursing Science, University of California, Irvine, CA, USA

${ }^{\mathrm{e}}$ Corresponding Author: Jill Howie-Esquivel, School of Nursing, University of California, \#2 Koret Way, Box 0610, San Francisco, CA 94143, USA. Email: jill.howie-esquivel@nursing.ucsf.edu

doi: http://dx.doi.org/10.14740/cr346w care and knowledge scores significantly improved $(\mathrm{P}<0.04$ and $\mathrm{P}$ $<0.02$, respectively) in the intervention group compared to UC.

Conclusion: The intervention utilized in Project Fluido was a remarkably effective method to improve self-care and HF knowledge in a group of Spanish-speaking HF patients. This improvement is in spite of low physical function, health literacy, acculturation and economic challenges. In addition, teach-back was an effective teaching strategy to improve HF knowledge. Future work is needed to investigate the relationship between increased self-care knowledge, readmissions, and mortality in Spanish-speaking patients with HF.

Keywords: Heart failure; Self-care; Hispanic; Health literacy; Patient knowledge

\section{Introduction}

Heart failure (HF) is a significant public health problem that affects 5.7 million people in the United States [1]. This problem is magnified in the Hispanic population because of the well-documented disparities in health care and the disproportionate cardiometabolic risk burden borne by this population $[2,3]$. Outcomes such as rehospitalization rates for Hispanic patients with HF are higher than in Caucasian populations [1]. The language barrier is a significant health care impediment, with over 8 million Hispanics living in the US who do not speak English fluently [4]. Monolingual Spanish speakers have a higher prevalence of cardiovascular risk factors and poorer recognition of cardiac symptoms compared to Hispanics who have English fluency [5].

A review of multiethnic clinical trials conducted in the US between 1995 and 1999 [6] found that Hispanics constituted only $3 \%$ of participants yet representing $15 \%$ of the general population [7]. Data demonstrating that Hispanics are as willing as non-Hispanic whites to participate in clinical trials suggest that their under-representation in clinical trials is less likely a result of reluctance to enroll, but rather inadequate access to research studies due to language barriers, inaccessibility to study sites and poor information dissemination [8]. 
Disease management programs focused on improving HF self-care are known to reduce hospitalization rates and mortality [9, 10]. Providing education that enables self/ family care is an essential first step for patients with HF. In fact, data demonstrate that the use of a psychoeducational intervention resulted in significantly better knowledge and self-care behavior in rural HF patients [11, 12]. However, few Hispanic patients participated in these studies in which bilingual staff was required. More importantly, the intervention would need to undergo a "cultural magnifying lens." In other words, the intervention would require consideration of Hispanic cultural values and modification to verify its appropriateness in the context of Hispanic culture. Further, a culturally appropriate intervention would allow examination of long-term outcomes such as rehospitalization and mortality.

In patients from non-English speaking backgrounds, consideration of health literacy is fundamental to improving health outcomes. A successful method for HF patient teaching, termed "teach-back," takes all literacy levels into consideration $[13,14]$. Teach-back was found to be a successful teaching method in a large group of hospitalized multi-ethnic HF patients [13]. No studies to date have been reported on the use of a culturally and health literacy-appropriate educational intervention exclusively for Spanish-speaking patients with HF. The potential for reducing health disparities among Spanish-speaking HF patients remains a significant opportunity for future research.

\section{Hypothesis}

We hypothesized that a culturally and health literacy-appropriate self-care educational intervention would improve selfcare behaviors and HF knowledge compared with a usual care (UC) group. In addition, we examined the relationship between psychosocial factors and the outcomes of self-care and HF knowledge.

\section{Methods}

\section{Research design}

The research design of Project Fluido was a prospective, pilot randomized controlled trial (RCT) that followed HF patients over 90 days. This study included two groups, an intervention and UC group with follow-up conducted at 3 months.

\section{Sample}

A convenience sample of 42 patients with HF were enrolled. Inclusion criteria were: 1) $\geq 18$ years of age, 2) hospitalized for $\mathrm{HF}$ within the previous 12 months, 3) identify themselves as Hispanic in origin, and 4) speak or write in English or
Spanish. We included patients who had been recently hospitalized as they are at higher risk for readmission. Exclusion criteria included: 1) cognitive impairment that limited ability to understand and complete questionnaires, 2) severe life-limiting co-morbidity (e.g. life expectancy of less than 6 months), and 3 ) live in a nursing home (could not participate in self-care activities).

\section{Procedure}

The study was approved by the Institutional Review Board at the University of California, San Francisco (UCSF), Research Committee at San Francisco General Hospital (SFGH) and nationally registered (NCT02083744). Recruitment took place at both UCSF and SFGH between April 2012 and March 2013 until the final sample size was obtained. Eligible patients were first introduced to the research study by other healthcare providers (cardiologists or nurse practitioners), and then interested patients were provided details of the study and enrolled by the research nurse. Patients who presented to the cardiology clinic or were being discharged from the hospital were recruited. The process of designing the study as culturally appropriate took place over 6 months prior to the initiation of the pilot study. Experts in HF care, Hispanic culture, lay people and nursing convened to design the study and materials with details described elsewhere.

After written consents were obtained, patients completed the Mini-Cog test for cognitive function to verify eligibility and completed baseline questionnaires [15]. The research nurse then opened sealed opaque envelopes that contained the assignment to the intervention or UC group. Due to the nature of the study design, the PI or the research nurse could not be blinded to the intervention once group assignment was determined.

Clinical data were collected from medical record review and patients completed the following questionnaires with the research nurse: 1) sociodemographic survey, 2) self-care heart failure index $[16,17], 3)$ brief symptom inventory subscale for anxiety [18], 4) patient health questionnaire-8 [19], 5) multi-dimensional scale for perceived social support [20], 6) short assessment of literacy for Spanish adults [21], and 7) the short acculturation scale for Hispanics [22].

The intervention consisted of five components (Table 1). The intervention group received a 1-on-1 educational session conducted by a bilingual/bicultural research nurse at the clinic site, the patients' home, or local cafe depending on patient preference. Patients received the educational intervention via a laptop computer and then teach-back questions were discussed (Table 1). To reinforce the educational intervention content a DVD, memory stick, or paper copy of the presentation was provided to the patient (whichever the patient preferred). Patients received a scale to measure weight, a diary to record weight and symptoms of HF, culturally appropriate no-salt spices and recipes, and telephone 
Table 1. Components of the Intervention

\author{
1-on-1 education \\ Scale, spices and recipes \\ Script \\ Diary/calendar \\ Telephone follow-up
}

Presentation on laptop \& written materials

Daily weights done upon awakening

No-salt, chili-based spices

Low-salt Latin-American recipes in large text format

Word for word description of how to talk with their health care provider in English and Spanish

Patients recorded daily weights and symptoms

Warning signs for action follow-up from the research nurse to reinforce the content of the education program every other week two additional times. The research nurse contacted patients every 2 weeks to: 1) reinforce the intervention and use of the diary, 2) discuss weights and symptoms, 3) discuss any unplanned healthcare visits, and 4) provide opportunity to ask questions. Patients were also given a script to use when calling their healthcare provider about increased HF symptoms in Spanish and English. The purpose of the script was to guide them in how to discuss their symptoms, if worse, with their healthcare provider. Patients were called close to the end of their 90-day participation to schedule a follow-up visit at which time four questionnaires (self-care heart failure index, brief symptom inventory subscale for anxiety, patient health questionnaire-8, multi-dimensional scale for perceived social support) were repeated.

Patients in the UC group were given a scale and the educational materials (without the 1-on-1 education). UC patients were not instructed on how to use the scale. Since most patients were from low socio-economic backgrounds, the researchers felt that it was unethical to withhold scales.
The UC group did not receive spices, recipes, script or nurse follow-up phone calls.

All patients were given a $\$ 50$ local grocery store gift card upon completion of the questionnaires. Clinical outcomes were examined in this study by capturing adverse events such as all cause morbidity and mortality within 90 days through electronic medical record review and from patient phone calls.

\section{Outcome measures: self-care and HF knowledge}

Self-care management skills were measured using the selfcare heart failure index $[16,17]$. This instrument is a 22-item well-validated instrument that measures self-care with three subscales: management, maintenance and confidence. The instrument was validated in Spanish speakers and items are scored in a 0 - 100 range [17].

The measurement of HF knowledge took place during the process of teach-back. Teach-back is a health-literacy appropriate method of patient teaching where the educator teaches the patient in plain language and then asks the pa-

Table 2. Teach-Back Questions

$\begin{array}{ll}\text { Teach-back question } & \text { Area of focus }\end{array}$

What is the name of your water pill?

How much weight gain would you want to report to your MD?

What high-salt foods do you need to avoid/be aware of?

Please name 3 - 4 symptoms or warning signs of when you want to call the MD?
Medications

Self-monitoring skills

Diet modification

Warning signs for action

At the conclusion of the teaching each patient was asked four teach-back questions. 


\section{Enrollment}

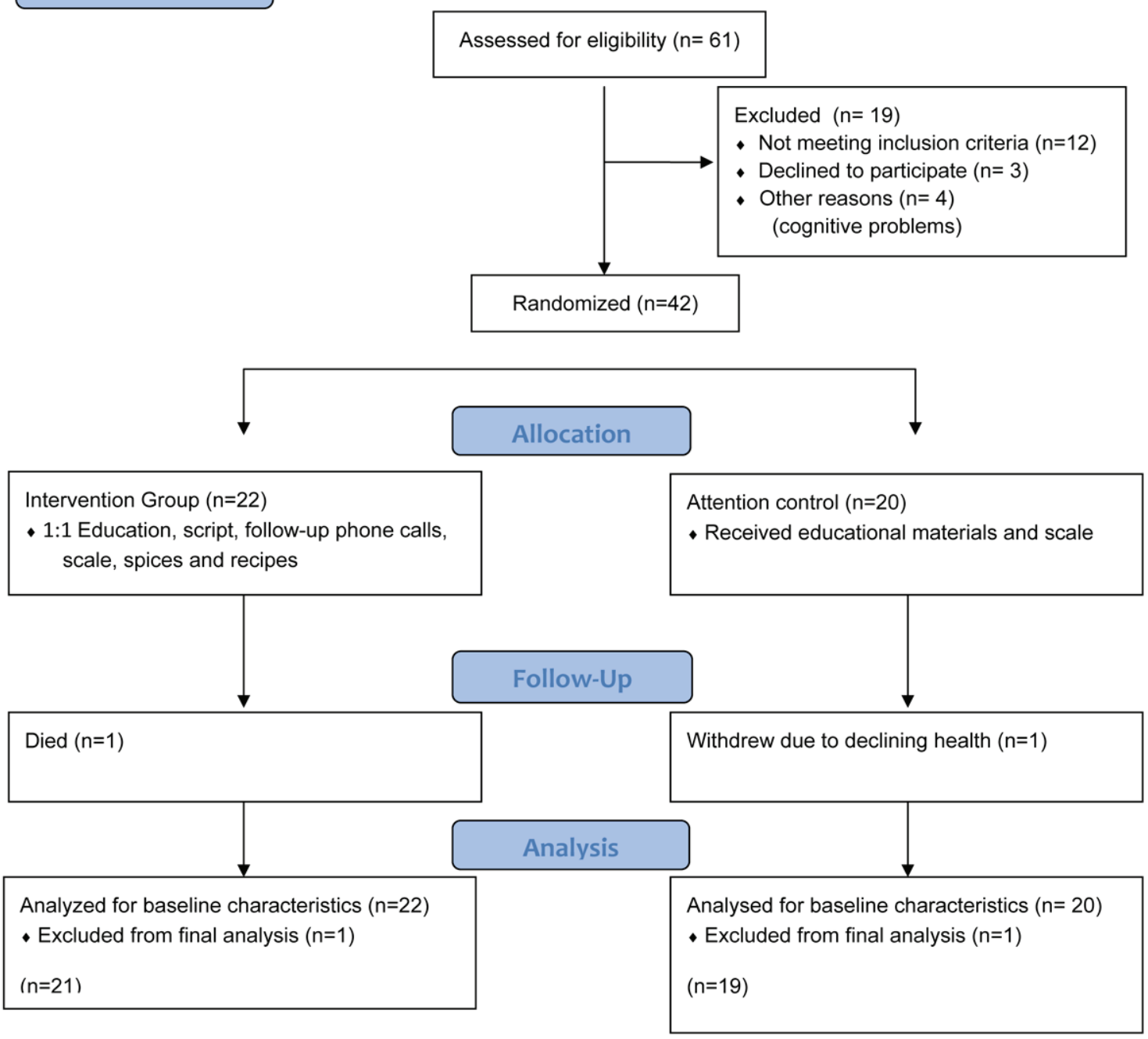

Figure 1. Patient screening and recruitment.

tient to teach-back the information that had been presented to them $[13,23]$. The concept underpinning teach-back education involves asking patients to restate information that has been presented to them. Teach-back education can serve as a method of education and a tool to assess learning.

HF-specific education was provided to the patients and included information related to: physiological function of the heart, rationale for fluid and sodium restrictions, importance of adherence to pharmacological therapies, rationale for weighing daily, signs and symptoms warranting provider notification, and how to talk to their provider. Patient's family members, caregivers, and/or support persons were also educated when available. Learning was assessed using four teach-back questions at the conclusion of the education session (Table 2). The four knowledge topics included educa- tional content related to: high salt foods, when to call their healthcare provider, when to report weight gain and the use of diuretics. Patients with incorrect responses were provided further education until understanding was achieved.

\section{Psychosocial and literacy measures}

Acculturation and health literacy were only measured at baseline because it was unlikely that they would change within 90 days. Acculturation was measured using the short acculturation scale for Hispanics that allows researchers to categorize study participants into high or low acculturation levels using four questions [22]. Validity and reliability are high and the scale has been tested in Mexican Americans, Puerto Ricans, Central and South Americans [22]. Health 
Table 3. Baseline Sociodemographic, Clinical and Psychological Characteristics $(N=42)$

\section{Usual care group $(n=20) \quad$ Intervention group $(n=22) \quad P$}

\begin{tabular}{|c|c|c|c|}
\hline Age, years (range $25-84$ ) & $53.7 \pm 12.3$ & $60.7 \pm 14.8$ & 0.11 \\
\hline Gender & & & 0.21 \\
\hline Female & $11(55.0)$ & $7(31.8)$ & \\
\hline Male & $9(45.0)$ & $15(68.2)$ & \\
\hline Low acculturation score ${ }^{a}$ & $13(41)$ & $9(60)$ & 0.10 \\
\hline Living arrangement & & & 0.39 \\
\hline Lives alone & $3(15.0)$ & $2(9.1)$ & \\
\hline Lives with $1-3$ others & $12(60.0)$ & $9(40.9)$ & \\
\hline Lives with 4 - 7 others & $3(15.0)$ & $8(36.4)$ & \\
\hline Lives with 8 or more & $2(10.0)$ & $3(13.6)$ & \\
\hline Annual salary & & & 0.23 \\
\hline$<20,000$ & $19(95.0)$ & $20(90.9)$ & \\
\hline $20,000-40,000$ & $0(0)$ & $2(9.1)$ & \\
\hline $40,000-75,000$ & $1(5.0)$ & $0(0)$ & \\
\hline Relationship status & & & 1.0 \\
\hline Married & $9(45.0)$ & $9(40.9)$ & \\
\hline Not-married & $11(55.0)$ & $13(55.1)$ & \\
\hline Sahlsa ${ }^{b}$ score & $40.4 \pm 6.7$ & $42 \pm 6.4$ & 0.40 \\
\hline \multicolumn{4}{|l|}{ Clinical characteristics } \\
\hline NYHA classification & & & 0.49 \\
\hline NYHA I & $0(0)$ & $1(4.5)$ & \\
\hline NYHA II & $5(25.0)$ & $9(40.9)$ & \\
\hline NYHA III & $12(60.0)$ & $10(45.5)$ & \\
\hline NYHA IV & $3(15.0)$ & $2(9.1)$ & \\
\hline Hypertension & $12(60.0)$ & $15(68.2)$ & 0.75 \\
\hline Diabetes & $8(40.0)$ & $10(45.5)$ & 0.66 \\
\hline Ejection fraction $<40 \%$ & $6(30.0)$ & $8(40.0)$ & 0.74 \\
\hline Taking ACE-I & $11(45.8)$ & $13(54.2)$ & 1.0 \\
\hline Taking ARB & $1(5.0)$ & $3(13.6)$ & 0.60 \\
\hline \multicolumn{4}{|l|}{ Psychosocial characteristics } \\
\hline Depression $^{\mathrm{c}}$ & $10.8 \pm 5.89$ & $6.73 \pm 5.85$ & 0.03 \\
\hline Anxiety $^{\mathrm{d}}$ & $1.13 \pm 1.09$ & $0.62 \pm 0.74$ & 0.09 \\
\hline Social support ${ }^{\mathrm{e}}$ & $64.7 \pm 14.5$ & $64.1 \pm 13.1$ & 0.88 \\
\hline
\end{tabular}

Data are presented as mean $\pm \mathrm{SD}, \mathrm{n}(\%), \mathrm{P}<0.05$. aBased on short acculturation score for Hispanics (less than 2.99 is less acculturated). 'bahlsa, short assessment of health literacy for Spanish adults. 'Based on patient health questionnaire-8 (range $0-24,10$ or greater indicates depression). ${ }^{\mathrm{d} B a s e d}$ on brief symptom inventory subscale for anxiety (range $0-4$, higher score indicates anxiety). ${ }^{e}$ Based on multi-dimensional scale of social support (range $7-84,69-84$ indicates high perceived levels of support). NYHA: New York Heart Association classification (range I - IV); ACE-I: angiotensin converting enzyme-inhibitor; ARB: angiotensin receptor blocker. 
literacy was measured using the short assessment of health literacy for Spanish-speaking adults (SAHLSA-50), which incorporates word recognition and comprehension using multiple choice questions [20]. This valid and reliable instrument was found to be associated with physical health in Spanish-speaking adults [20].

Depression was measured with the eight-item personal health questionnaire- 8 , a brief and valid tool used for depression screening [19]. Scores range from 0 to 24 and a score of 10 or greater suggests clinical depression. The personal health questionnaire- 8 has well-established validity, sensitivity and specificity [19]. Anxiety was measured using the six-item anxiety subscale of the brief symptom inventory and measures state anxiety [18]. Scores range from 0 to 4 and the overall score is the average score of all items with higher scores indicating higher anxiety. It has demonstrated reliability and validity in cardiac patients [18]. Social support was measured using the validated 12-item multidimensional scale of perceived social support [20]. Perceptions about support from family, friends and a significant other were assessed with scores ranging from 7 to 84 where higher scores indicated higher levels of perceived support.

\section{Statistical analysis}

Descriptive statistics, means and standard deviations (SDs) for continuous variables, and frequencies and percentages for categorical variables were provided for all sociodemographic and clinical variables. Study group differences in sociodemographic and clinical characteristics were examined with Chi-square and Student's $t$-tests analyses. All data were analyzed with intention to treat. Missing data were assumed to be missing completely at random. SPSS 21.0 was used for all analyses.

In order to estimate the necessary sample size, power analyses were performed utilizing data from a previous study [9]. It was hypothesized that the intervention group would show more improvement in HF self-care behavior and HF knowledge than the UC group. For self-care, which was the outcome determining sample size for this pilot study, the difference between the intervention and control groups was 1.0 with a common SD of 1.15, therefore the estimated effect size was $d=0.87$. Based on an estimate of the effect size being at least $d=0.87$, in order to achieve power of 0.80 at an alpha level of $0.05,22$ subjects per group (total sample of 44) were needed to find statistical significance.

To compare the effect of the intervention to UC on HF knowledge and self-care behaviors, a repeated measures analysis was used with a linear mixed models approach. This analysis allows for testing the main effect of group, main effect of time and the group-by-time interaction. Linear regression was used to examine predictors of self-care and teachback knowledge. Significance was defined as $\mathrm{P}<0.05$ and data are presented as means \pm SDs where appropriate.

\section{Results}

\section{Characteristics of study participants}

Forty-two patients were recruited to participate and completed baseline questionnaires; however, one patient withdrew and one died before the study end. Figure 1 displays patient screening and recruitment according to the CONSORT guidelines [24]. No differences in sociodemographic or clinical characteristics (including ED or hospitalizations) were found among groups except with depression levels, where the UC group had higher baseline levels of depression (Table 3 ). Overall, the sample mean age was $57 \pm 14$ years (range 25 - 84), 57\% were male, and 57\% unmarried. Poor health literacy was present in $31 \%$, low acculturation scores in $52 \%$, and $67 \%$ spoke Spanish only. Financial resources were limited with $93 \%$ of participants who reported an income of $<$ $\$ 20,000$ and 38\% lived with four or more in the home.

Clinical characteristics included 64\% who had hypertension, 64\% were New York Heart Association (NYHA) Class III/IV and $65 \%$ had HF with reduced ejection fraction. Psychosocial measures revealed mean baseline depression scores of $8.6 \pm 6.15$ (range $0-27$ where depression is present when scores are $\geq 10$ ), mean anxiety scores of $0.87 \pm 0.95$ (range 0 - 4 where higher scores indicate more anxiety) and social support scores of $64 \pm 14$ (range 7 - 84 where higher scores indicate higher levels of perceived support). Both the intervention and UC groups did experience improvements in depression and anxiety scores but the differences were not significant (Table 4).

\section{Self-care and knowledge}

Mean baseline self-care management scores were $56 \pm 23$, self-care maintenance $60 \pm 18$ and confidence $71 \pm 20$ (all subscales range 0 - 100 with higher scores indicating better self-care). In the intervention group, self-care management scores significantly improved from a mean of 49 to $81(\mathrm{P}=$ 0.02). Self-care maintenance improved in both groups, but was not statistically different. Self-care confidence did not improve. Anxiety was found to be a predictor of self-care maintenance ( $\mathrm{P}=0.03, \mathrm{CI}:-15.5,-0.84)$, while depression and social support were not. For every one-unit increase in anxiety, there was an eight-point decrease in self-care management (Table 5). Depression scores did improve in both groups; however, baseline scores were significantly different between the groups (higher in UC).

Mean baseline teach-back scores were 3.69+0.51 (range 0 - 4 with higher scores indicating better knowledge). In the intervention group, teach-back scores significantly improved from a mean of 3.6 to $4.0(\mathrm{P}=0.04)$. Mean time spent teaching the intervention group was $78 \pm 18 \mathrm{~min}$. There were no significant psychosocial predictors of teach-back scores. The effect size of the intervention for comparing the intervention 
Table 4. Self-Care, Teach-Back and Psychosocial Scores by Group

\begin{tabular}{|c|c|c|c|}
\hline & $\begin{array}{l}\text { Usual care group (n } \\
=20 \text { ) }\end{array}$ & $\begin{array}{l}\text { Intervention group ( } \\
=22)\end{array}$ & $\mathbf{P}$ \\
\hline Teach-back scores & & & 0.04 \\
\hline Mean & 3.8 & 3.6 & \\
\hline Baseline & 3.9 & 4.0 & \\
\hline Final range & $(3.8-3.9)$ & $(3.9-4.1)$ & \\
\hline Self-care management ${ }^{\mathrm{a}}$ & & & 0.02 \\
\hline Mean & 58.9 & 49.2 & \\
\hline Baseline & 58.0 & 81.0 & \\
\hline Final & $(41.9-74.2)$ & $(62.7-99.4)$ & \\
\hline Self-care maintenance ${ }^{a}$ & & & 0.88 \\
\hline Mean & 56.8 & 63.6 & \\
\hline Baseline & 70.0 & 77.7 & \\
\hline Final & $(61.3-78.6)$ & $(69.1-86.1)$ & \\
\hline Self-care confidence ${ }^{a}$ & & & 0.79 \\
\hline Mean & 68.5 & 73.5 & \\
\hline Baseline & 70.8 & 74.0 & \\
\hline Final & $(61.8-80.3)$ & $(64.7-83.4)$ & \\
\hline Depression $^{\mathrm{b}}$ & & & 0.69 \\
\hline Mean & 10.8 & 6.7 & \\
\hline Baseline & 9.3 & 6.1 & \\
\hline Final & $(5.7-12.8)$ & $(2.6-9.6)$ & \\
\hline Anxiety $^{\mathrm{c}}$ & & & 0.26 \\
\hline Mean & 1.13 & 0.62 & \\
\hline Baseline & 0.83 & 0.71 & \\
\hline Final & $(0.35-1.3)$ & $(0.23-1.9)$ & \\
\hline Social support ${ }^{d}$ & & & 0.57 \\
\hline Mean & 64.7 & 64.1 & \\
\hline Baseline & 64.2 & 65.6 & \\
\hline Final & $(56.8-71.5)$ & $(58.5-72.8)$ & \\
\hline
\end{tabular}

${ }^{\mathrm{a} B}$ Based on the self-care heart failure index. ${ }^{\mathrm{b}}$ Based on patient health questionnaire-8. ${ }^{\mathrm{c} B a s e d}$ on brief symptom inventory subscale for anxiety. ${ }^{d B}$ Based on multidimensional scale of social support. Cl: confidence interval.

to UC group was calculated on self-care scores. There was a large effect size with Cohen's $d=0.89$.

\section{Discussion}

These pilot data demonstrate that a culturally appropriate ed- ucational intervention improved self-care behaviors and HF knowledge in comparison to a UC group in the first study of its kind. In addition, anxiety was a predictor for self-care management.

Few studies address the association between minority status and self-care management in cardiovascular disease. One group of investigators examined the association be- 
Table 5. Psychosocial Predictors of Self-Care and Heart Failure Knowledge $(\mathrm{N}=42)$

\begin{tabular}{lllll}
\hline Baseline & SE & $\mathbf{P}$ & Exponent B & $\mathbf{9 5 \%}$ CI \\
\hline $\begin{array}{l}\text { Self-care maintenance } \\
\quad\end{array}$ & & & & \\
Depression $^{\mathrm{b}}$ & 0.56 & 0.58 & 0.32 & $-0.82,1.45$ \\
Anxiety $^{\mathrm{c}}$ & 3.63 & 0.03 & -8.20 & $-15.5,-0.84$ \\
Social support $^{\mathrm{d}}$ & 0.19 & 0.08 & 0.35 & $-0.06,0.75$ \\
Teach-back knowledge $^{\text {Depression }}$ & & & & $-0.02,0.05$ \\
Anxiety & 0.017 & 0.284 & 0.018 & $-0.33,0.11$ \\
Social support & 0.108 & 0.327 & -0.107 & $-0.00,0.02$ \\
\hline
\end{tabular}

SEM: standard error of mean $(\mathrm{P}<0.05)$. Cl: confidence interval. ${ }^{a}$ Based on self-care heart failure index. ${ }^{b}$ Based on patient health questionnaire-8 depression instrument. 'Based on brief symptom inventory subscale for anxiety instrument. ${ }^{\mathrm{d} B a s e d}$ on multi-dimensional scale of social support instrument.

tween minority status and self-care confidence in recently hospitalized cardiac patients [25]. Hispanics had the lowest confidence levels to self-manage in comparison to black or white patients. However, when health status and socioeconomic circumstances were controlled for, the association was no longer apparent, thus illustrating the importance of clinical and economic conditions on health. In our study, we measured self-care confidence and found that our intervention did not significantly increase self-confidence. However, we found that self-care management did increase, calling into question the exact relationship between self-care confidence and self-care management. If self-care confidence skills increase, do self-care management skills, or the behaviors of self-care change?

Caldwell and colleagues tested a similar educational intervention in a small group of rural HF patients and found that self-care and HF knowledge improved [11]. In a large group of rural HF patients, investigators found improvements in self-care, HF knowledge and cardiac mortality after testing an educational intervention in two doses, LITE and PLUS where the LITE group demonstrated improved cardiac mortality [12]. Our intervention key elements (education, scale, script and nurse phone calls) were similar to the LITE group and our results are comparable although our study was not powered to test outcomes such as mortality and hospitalizations.

Despite the numerous endorsements from national organizations such as the Heart Failure Society of America to provide patient education, no studies have been conducted to compare the efficacy of different patient teaching meth- ods. Several investigators have demonstrated that teachback is an effective strategy for patient teaching $[13,14]$. However, few studies have linked patient teaching methods to improved patient outcomes. A health literacy sensitive approach to patient education demonstrated improvement in HgA1c levels in one small study [23]. In the only RCT that tested teach-back on patient outcomes known to the authors, asthmatic patients had improved asthma knowledge and fewer 30-day acute health-related events [26]. In our study teach-back was associated with improved HF knowledge, although our sample size was not large enough to test clinical outcomes.

Psychological symptoms such as depression have been found to correlate with low self-efficacy in chronic illness self-management interventions [27]. Sin and colleagues assessed the prevalence and personal characteristics that predict depressive symptoms in Hispanics with HF after a telephone case management intervention [28]. Depressive symptoms were highly prevalent and acculturation, co-morbidity and NYHA class were significantly related to depressive symptoms. In our study, depression symptoms improved in both the control and the intervention groups. The cause of the improvement in symptoms is unclear, but may be related to the Hawthorne effect. Anxiety, but not depression significantly predicted self-care management scores. It is known that HF patients experience high levels of anxiety [29], but no data exist to describe anxiety in Hispanic patients with HF. Riegel and colleagues investigated the effect of a 6-month telephone case management program on hospital readmissions and depression in one of the few disease management studies that 
enrolled Hispanics of Mexican-American origin [30]. The authors found no improvement in HF readmissions, mortality or depression. This study did not include an educational or cultural component to the intervention and a subsequent editorial opined that HF knowledge and patient beliefs may have played a role in this negative study [31].

Limitations may apply in this pilot RCT. First, the small convenience sample may have undermined the external validity of the findings. The sample was predominately male, low-income and with low health literacy. The results may be different in women and populations reflecting greater income and education. Second, the follow-up period of 90 days did not allow for long-term adherence trends or clinical outcomes, particularly given the setting of a chronic disease such as HF. The Hawthorne effect may have been present in this study, especially within the UC group since they received scales and patient education handouts. The scales and handouts may have added unintentional attention that resulted in changes in anxiety and depression scores.

Despite the potential limitations, several positive attributes of this study are present. No research to date regarding culturally appropriate interventions for the largest ethnic group in the US has been previously reported. The feasibility of this intervention has been established with a low attrition rate, and may be generalizable to a wider population. Future research in disease management with underserved populations will help determine the appropriate dosing and content of education and follow-up through long-term studies. Improvements in self-care and knowledge are needed to correspond to long-term clinical outcomes such as rehospitalization and mortality. As our population continues to diversify in ethnicity, race, sex and age, studies are needed to address the key aspects of self-care skill building and patient education.

\section{Conclusion}

Project Fluido was an effective and culturally appropriate intervention to improve self-care and HF knowledge in a group of Spanish-speaking HF patients. The improvement was achieved despite low physical function, poor health literacy, acculturation and economic challenges faced by the participants. In addition, teach-back was an effective teaching strategy to improve HF knowledge. Future work is needed to investigate the relationship between increased self-care knowledge and hospital readmissions and mortality in Spanish-speaking patients with HF.

\section{Acknowledgement}

We would like to thank Zenelia Roman RN, MS for her support in recruitment and patient teaching and Steven Paul, $\mathrm{PhD}$, biostatistician at UCSF, for his expert guidance in the statistical analyses of this study.

\section{Funding}

Funding for research was provided by a grant from the Robert Wood Johnson Foundation.

\section{Disclosures}

The authors have no disclosures.

\section{References}

1. Go AS, Mozaffarian D, Roger VL, Benjamin EJ, Berry JD, Blaha MJ, Dai S, et al. American Heart Association Statistics Committee and Stroke Statistics Subcommittee. Heart disease and stroke statistics 2014 update: A report from the American Heart Association. Circulation. 2014;129:e242-e247.

2. Weinick RM, Jacobs EA, Stone LC, Ortega AN, Burstin $\mathrm{H}$. Hispanic healthcare disparities: challenging the myth of a monolithic Hispanic population. Med Care. 2004;42(4):313-320.

3. Go AS, Mozaffarian D, Roger VL, Benjamin EJ, Berry JD, Blaha MJ, Dai S, et al. American Heart Association Statistics Committee and Stroke Statistics Subcommittee. Heart disease and stroke statistics 2014 update: A report from the American Heart Association. Circulation.2014;129(3):e60-96.

4. Institute of Medicine. The health, health insurance and language status of racial and ethnic minority populations. In: Smedley BD, Stith AY, Nelson AR, editors. Unequal Treatment: Confronting Racial and Ethnic Disparities in Health Care. Washington DC: National Academes Press. 2003:88-89.

5. DuBard CA, Garrett J, Gizlice Z. Effect of language on heart attack and stroke awareness among U.S. Hispanics. Am J Prev Med. 2006;30(3):189-196.

6. Evelyn B, Toigo T, Banks D, Pohl D, Gray K, Robins B, Ernat J. Participation of racial/ethnic groups in clinical trials and race-related labeling: a review of new molecular entities approved 1995-1999. J Natl Med Assoc. 2001;93(12 Suppl):18S-24S.

7. US Census Bureau. People Quickfacts. http://quickfacts. census.gov/qfd/states/00000.html. Accessed June 25, 2014.

8. Vivo RP, Krim SR, Cevik C, Witteles RM. Heart failure in Hispanics. J Am Coll Cardiol. 2009;53(14):11671175 .

9. McAlister FA, Stewart S, Ferrua S, McMurray JJ. Multidisciplinary strategies for the management of heart 
failure patients at high risk for admission: a systematic review of randomized trials. J Am Coll Cardiol. 2004;44(4):810-819.

10. Gohler A, Januzzi JL, Worrell SS, Osterziel KJ, Gazelle GS, Dietz R, Siebert U. A systematic meta-analysis of the efficacy and heterogeneity of disease management programs in congestive heart failure. J Card Fail. 2006;12(7):554-567.

11. Caldwell MA, Peters KJ, Dracup KA. A simplified education program improves knowledge, self-care behavior, and disease severity in heart failure patients in rural settings. Am Heart J. 2005;150(5):983.

12. Dracup K, Moser DK, Pelter MM, Nesbitt TS, Southard J, Paul SM, Robinson S, et al. Randomized, controlled trial to improve self-care in patients with heart failure living in rural areas. Circulation. 2014;130(3):256-264.

13. White M, Garbez R, Carroll M, Brinker E, Howie-Esquivel J. Is "teach-back" associated with knowledge retention and hospital readmission in hospitalized heart failure patients? J Cardiovasc Nurs. 2013;28(2):137-146.

14. Wilson FL, Mayeta-Peart A, Parada-Webster L, Nordstrom C. Using the teach-back method to increase maternal immunization literacy among low-income pregnant women in Jamaica: a pilot study. J Pediatr Nurs. 2012;27(5):451-459.

15. Carolan Doerflinger DM. How to try this: the mini-cog. Am J Nurs. 2007;107(12):62-71; quiz 71-62.

16. Riegel B, Carlson B, Glaser D. Development and testing of a clinical tool measuring self-management of heart failure. Heart Lung. 2000;29(1):4-15.

17. Vellone E, Riegel B, Cocchieri A, Barbaranelli C, D’Agostino F, Antonetti G, Glaser D, et al. Psychometric testing of the Self-Care of Heart Failure Index Version 6.2. Res Nurs Health. 2013;36(5):500-511.

18. Abu Ruz ME, Lennie TA, Riegel B, McKinley S, Doering LV, Moser DK. Evidence that the brief symptom inventory can be used to measure anxiety quickly and reliably in patients hospitalized for acute myocardial infarction. J Cardiovasc Nurs. 2010;25(2):117-123.

19. Kroenke K, Spitzer RL, Williams JB. The PHQ-9: validity of a brief depression severity measure. J Gen Intern Med. 2001;16(9):606-613.

20. Zimet GD, Powell SS, Farley GK, Werkman S, Berkoff KA. Psychometric characteristics of the Multidimen- sional Scale of Perceived Social Support. J Pers Assess. 1990;55(3-4):610-617.

21. Lee SY, Bender DE, Ruiz RE, Cho YI. Development of an easy-to-use Spanish Health Literacy test. Health Serv Res. 2006;41(4 Pt 1):1392-1412.

22. Marin G, Sabogal F, VanOss Marin B, Otero-Sabogal F, Perez-Stable EJ. Development of a short acculturation scale for Hispanics. Hispanic Journal of Behavioral Sciences. 1987;9:183-205.

23. Schillinger D, Piette J, Grumbach K, Wang F, Wilson C, Daher C, Leong-Grotz K, et al. Closing the loop: physician communication with diabetic patients who have low health literacy. Arch Intern Med. 2003;163(1):8390.

24. Schulz KF, Altman DG, Moher D, Group C. CONSORT 2010 statement: updated guidelines for reporting parallel group randomised trials. PLoS Med. 2010;7(3):e1000251.

25. Blustein J, Valentine M, Mead H, Regenstein M. Race/ Ethnicity and patient confidence to self-manage cardiovascular disease. Med Care. 2008;46(9):924-929.

26. Press VG, Arora VM, Shah LM, Lewis SL, Charbeneau J, Naureckas ET, Krishnan JA. Teaching the use of respiratory inhalers to hospitalized patients with asthma or COPD: a randomized trial. J Gen Intern Med. 2012;27(10):1317-1325.

27. Hwang B, Moser DK, Dracup K. Knowledge is insufficient for self-care among heart failure patients with psychological distress. Health Psychol. 2014;33(7):588596.

28. Sin MK. Personal characteristics predictive of depressive symptoms in Hispanics with heart failure. Issues Ment Health Nurs. 2012;33(8):522-527.

29. Suzuki T, Shiga T, Kuwahara K, Kobayashi S, Suzuki S, Nishimura K, Suzuki A, et al. Impact of clustered depression and anxiety on mortality and rehospitalization in patients with heart failure. J Cardiol. 2014.

30. Riegel B, Carlson B, Glaser D, Romero T. Randomized controlled trial of telephone case management in Hispanics of Mexican origin with heart failure. J Card Fail. 2006;12(3):211-219.

31. Jaarsma T, van der Wal MH, van Veldhuisen DJ. Diversity in management of heart failure patients. J Card Fail. 2006;12(7):584-585. 